\title{
A Deconvolution Approach to the Three Dimensional Identification of Cracks in Magnetic Slabs
}

\author{
Amr A. Adly ${ }^{1}$, Salwa K. Abd-El-Hafiz ${ }^{2}$ \\ ${ }^{1}$ Departement of Electrical Power and Machines, Faculty of Engineering, Cairo University, Egypt \\ ${ }^{2}$ Departement of Engineering Mathematics, Faculty of Engineering, Cairo University, Egypt
}

\begin{tabular}{l} 
Article Info \\
\hline Article history: \\
Received Oct 17, 2016 \\
Revised Dec 4, 2016 \\
Accepted Feb 2, 2017 \\
\hline Keywords: \\
Crack detection \\
Deconvolution \\
Fourier transform \\
Magnetic analysis \\
Nondestructive testing \\
\hline
\end{tabular}

\begin{abstract}
Nondestructive assessment of cracks in metallic parts has always been a topic of industrial interest. In the past, different approaches have been proposed to assess such cracks. Recently, semi-orthogonal compactly supported spline wavelets were utilized to efficiently identify the 3D spatial location of cracks in conducting slabs of finite thicknesses. Within this proposed approach a horizontally oriented field sensor is employed on top of a magnetic slab subject to uniform horizontal magnetic field. In this paper the 3D spatial identification of cracks in conducting slabs is carried out through the Fourier transform by de-convoluting sensor response. In comparison to the previously adopted approach, the approach proposed in this paper is capable of identifying cracks that span over a relatively larger distance. Details of the crack detection methodology and simulations are given in the paper.
\end{abstract}

Copyright () 2017 Institute of Advanced Engineering and Science. All rights reserved.

\section{Corresponding Author:}

Amr A Adly,

Departement of Electrical Power and Machines,

Faculty of Engineering, Cairo University,

Giza 12613, Egypt.

Email: amradly@cu.edu.eg

\section{INTRODUCTION}

Spatial identification of crack locations in conducting slabs has been a topic of wide industrial interest. In the past, several nondestructive crack detection techniques have been introduced (see, for example, [1-5]). Among those techniques, stray magnetic field monitoring, as a result of impressed current crack perturbation, has proven to be successful $[6,7]$. Recently, the wavelets approach has been utilized in surface crack detection using impressed current differential measurements [8,9]. In this approach, semiorthogonal compactly supported spline wavelets were used to efficiently solve the resulting 2D Fredholm integral equation of the first kind and, finally, identify the 3D spatial location of cracks in conducting slabs of finite thicknesses.

While the wavelets approach proved successful, it offered discrete crack identification within the mesh discretization scheme. This paper presents a methodology for assessing cracks extending over relatively large dimensions in conducting slabs. The approach utilizes techniques proposed in [10-12] and employs the Fourier transform (FT) to de-convolute Gaussmeter finite sensor device response. As in [3], the approach under consideration employs a horizontally oriented field sensor placed on top of the magnetic slab under consideration while being subjected to a uniform horizontal magnetic field as shown in Fig. 1. In the presence of cracks, localized magnetic dipoles are formed, thus emanating vertical fields that could be detected by the sensor. Thus, a 2D magnetic field mapping resulting from the existence of cracks may be deduced. Details of the proposed methodology are given in the following section. 


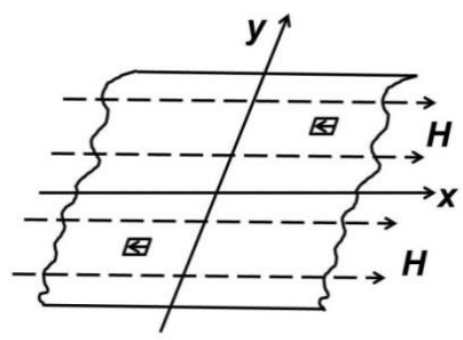

Figure 1. Localized magnetic dipole formation due to the existence of cracks in a conducting magnetic slab subject to uniform magnetic field.

\section{THE PROPOSED APPROACH}

Explaining It can be shown that the vertical magnetic flux density component $B_{z}$ resulting from an isolated magnetic charge $q_{m}$ may be deduced from the expression:

$$
B_{z}(x, y, z)=\frac{\mu_{O}\left(z-z_{S}\right) q_{m}\left(x_{s}, y_{s}, z_{S}\right)}{4 \pi\left[\left(x-x_{S}\right)^{2}+\left(y-y_{s}\right)^{2}+\left(z-z_{s}\right)^{2}\right]^{3 / 2}},
$$

where, $(x, y, z)$ is the observation point, $\left(x_{S}, y_{S}, z_{S}\right)$ is the magnetic charge position, while $\mu_{O}$ is the permeability of free space.

Assuming a square horizontal flux density sensor having side length and vertical height coordinate $H$ and $z_{O}$, respectively, it can be shown that - when its center coordinates coincide with $(x, y, z)-$ the measured flux density due to a unit magnetic charge (i.e., device response) may be given by:

$$
K_{z}\left(x, y, z_{o} ; x_{s}, y_{s}, z_{s}\right)=\frac{\mu_{O}\left(z_{o}-z_{S}\right)}{4 \pi L^{2}} \int_{(y-H / 2)}^{(y+H / 2)} \int_{(x-H / 2)}^{(x+H / 2)} \frac{1}{\left|\bar{R}_{P Q}\right|^{3 / 2}} d x^{\prime} d y^{\prime} .
$$

In (2), $\bar{R}_{P Q}=\left(x-x_{S}\right) \bar{u}_{x}+\left(y-y_{S}\right) \bar{u}_{y}+\left(z_{O}-z_{S}\right) \bar{u}_{z}$, while $\bar{u}_{x}, \bar{u}_{y}$ and $\bar{u}_{z}$ represent the unit vectors along the $\mathrm{x}$-, $\mathrm{y}$ - and $\mathrm{z}$-directions, respectively.

For a normalized square inspection zone far enough from the sides of a relatively large slab to ignore edge effects, it can be shown that the vertical flux density mapping $B_{z}^{M}$ inferred by the abovedescribed sensing element may be expressed by:

$$
B_{z}^{M}\left(x, y, z_{o}\right)=\int_{0}^{1} \int_{0}^{1} q_{m}\left(x_{s}, y_{s}, z_{S}\right) K_{Z}\left(x^{\prime}, y^{\prime}, z_{o} ; x_{S}, y_{S}, z_{s}\right) d x^{\prime} d y^{\prime} .
$$

Applying FT and the convolution principle, we get,

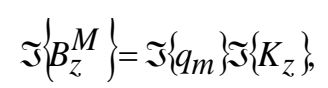

where $\mathfrak{I}$ is the Fourier transform. Hence, knowing the measurements profile as well as the device response, the dipole charge density distribution may be deduced using the inverse Fourier transform (IFT), $\mathfrak{J}^{-1}$, given by:

$$
q_{m}(x, y, z)=\mathfrak{I}^{-1}\left\{\frac{\mathfrak{I}\left\{B_{z}^{M}\right\}}{\mathfrak{J}\left\{K_{z}\right\}}\right\} .
$$
inferred.

Once the dipole charge distribution map is deduced, crack locations and dimensions may be 


\section{SAMPLE CRACK EVALUATION COMPUTATIONS}

Using the proposed methodology, computations have been carried out to identify cracks corresponding to different cases. Throughout the simulations, raw measured vertical flux density mapping $B_{z}^{M}$ was computed in accordance with equation (3) for cracks having a depth equivalent to $2 \%$ of the observation domain side length. More specifically, the eight crack cases given in Table 1 have been considered. It should be stated that, within the simulations, the observation domain was discretized into 61 by 61 mesh points and 2D FT was carried out up to the $41^{\text {st }}$ harmonic coefficient. It can be seen from the aforementioned table that the simulations cover sensor side lengths of $0.1,0.2,0.3$ and 0.5 measured in per unit $(\mathrm{pu})$ of observation domain side length.

Table 1. Crack identification cases considered.

\begin{tabular}{ccccc}
\hline CASE & $\begin{array}{c}\text { Vertical Clearance (pu } \\
\text { of domain side length) }\end{array}$ & $\begin{array}{c}\text { Sensor Side Length } H \\
\text { (pu of domain side length) }\end{array}$ & $\begin{array}{c}\text { Number } \\
\text { of Cracks }\end{array}$ & $\begin{array}{c}\text { Crack(s) Limiting Coordinates [lower left corner } \\
\text { - upper right corner] in mesh units }\end{array}$ \\
\hline 1 & 0.1 & 0.1 & 2 & {$[19,13-23,17] \&[39,45-43,49]$} \\
2 & 0.1 & 0.1 & 2 & {$[19,13-29,17] \&[39,45-43,49]$} \\
3 & 0.1 & 0.2 & 1 & {$[29,29-33,33]$} \\
4 & 0.2 & 0.2 & 1 & {$[29,29-33,33]$} \\
5 & 0.4 & 0.2 & 1 & {$[29,29-33,33]$} \\
6 & 0.1 & 0.2 & 2 & {$[19,13-23,17] \&[39,45-43,49]$} \\
7 & 0.1 & 0.3 & 1 & {$[29,29-33,33]$} \\
8 & 0.1 & 0.5 & 1 & {$[29,29-33,33]$} \\
\hline
\end{tabular}

Cases 1 and 2 correspond to a sensor side length of 0.1 pu. Figure 2 shows the device response $K_{z}$ for these two cases as well as the reconstructed response using the $41^{\text {st }}$ by $41^{\text {st }} 2 \mathrm{D} \mathrm{FT}$ harmonic coefficients. For these two cases, two cracks are assumed. More specifically, in Case 1 the two cracks are assumed to be of identical dimensions while for Case 2 they are assumed to be non-identical. In Figure 3 , computed $B_{z}^{M}$, actual cracks, apparent cracks deduced from raw image and inferred cracks according to equation (5) are shown for Case 1. Similar plots corresponding to Case 2 are given in Figure 4.

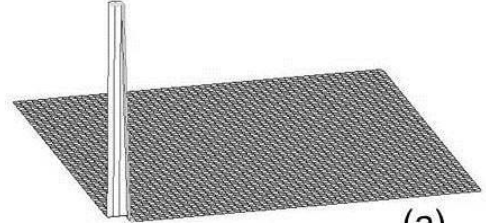

(a)

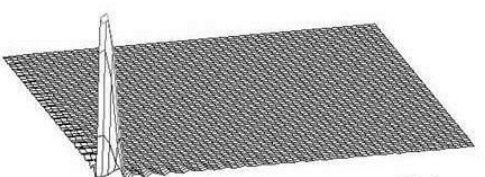

(b)

Figure 2. (a) The device response $K_{z}$ corresponding to Cases 1 and 2 and, (b) the reconstructed response using 2D FT harmonic coefficients.

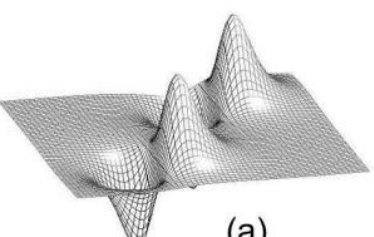

(a)

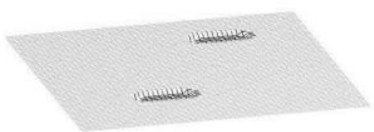

(c)

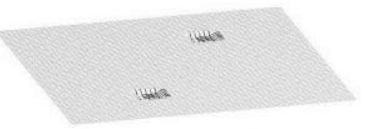

(b)

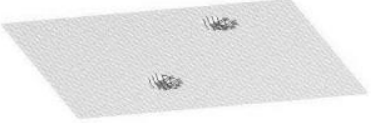

(d)

Figure 3. (a) Computed $B_{z}^{M}$, (b) actual cracks, (c) apparent cracks deduced from raw image, and (d) inferred cracks according to the proposed methodology for Case 1. 


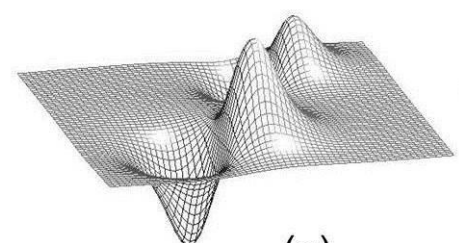

(a)

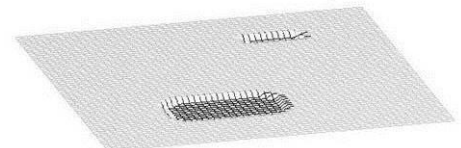

(c)

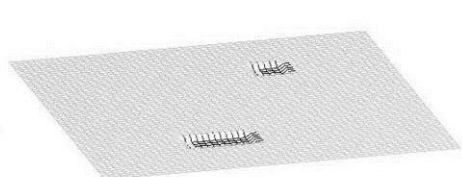

(b)

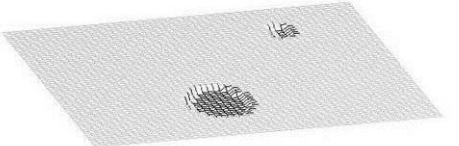

(d)

Figure 4. (a) Computed $B_{z}^{M}$, (b) actual cracks, (c) apparent cracks deduced from raw image, and (d) inferred cracks according to the proposed methodology for Case 2.

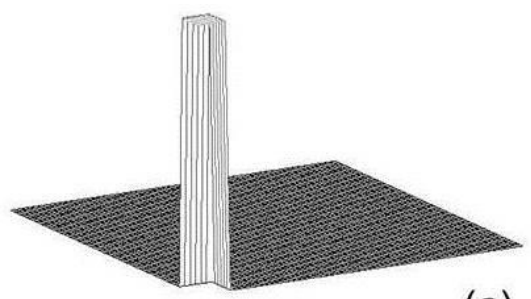

(a)

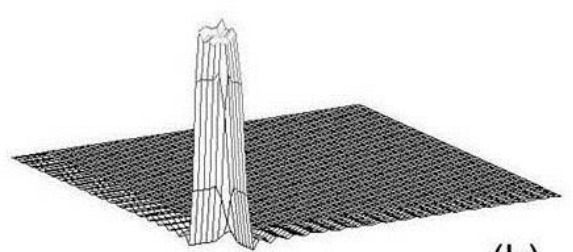

(b)

Figure 5. (a) The device response $K_{z}$ corresponding to Cases 3, 4 and 5 and (b) the reconstructed response using 2D FT harmonic coefficients. For Cases 3, 4, 5 and 6 a sensor side length of 0.2 pu is assumed. Figure 5 shows the device response $K_{z}$ for these four cases as well as its corresponding reconstructed response using the aforementioned 2D FT harmonic coefficients. Effect of having different sensor-sample clearance $z_{0}$ is clearly highlighted by Cases 3, 4 and 5. For these three cases, as suggested in Table I, the same crack is assumed for $z_{o}$ pu values of $0.1,0.2$ and 0.4 , respectively. Images of $B_{z}^{M}$ computed in accordance with equation (3) for those cases are given in Figure 6. Plots for the actual cracks, apparent cracks deduced from raw images and inferred cracks, according to equation (5), corresponding to the above-mentioned three cases are shown in Figure. 7-9. Case 6, on the other hand, corresponds to the same double cracks previously assumed for Case 1. In Figure 10, plots corresponding to those shown in Figure 3 are given, thus further highlighting the effect of sensor-sample clearance $z_{O}$ variation.

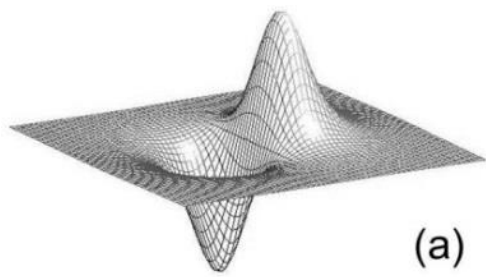

(a)

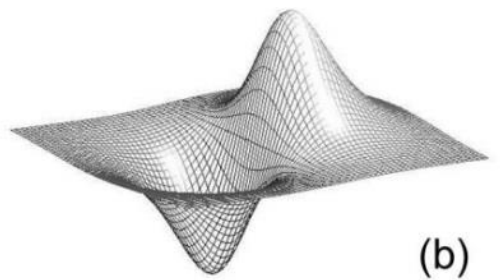

(b)

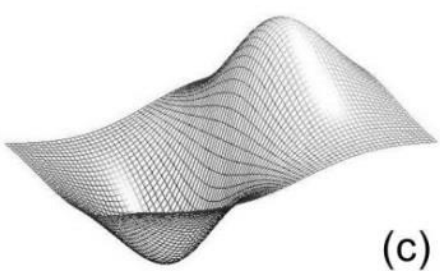

(c)

Figure 6. Computed $B_{z}^{M}$ corresponding to (a) Case 3, (b) Case 4, and (c) Case 5. 


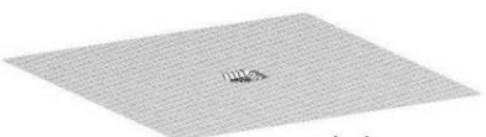

(a)

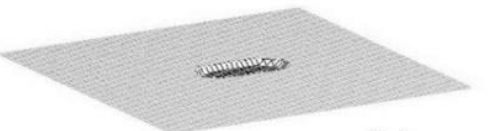

(b)

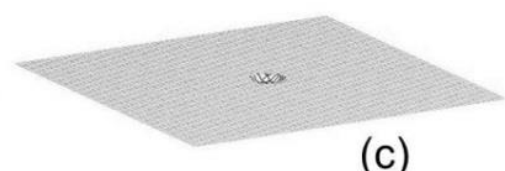

(c)

Figure 7. (a) Actual crack, (b) apparent crack deduced from raw image, and (c) inferred crack according to the proposed methodology for Case 3.

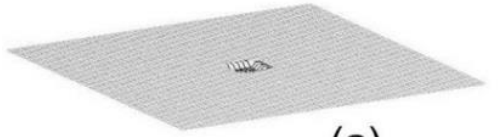

(a)

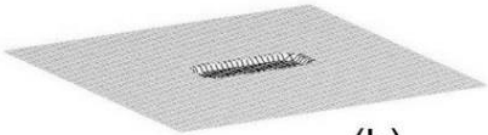

(b)

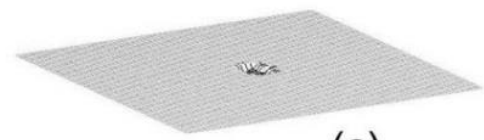

(c)

Figure 8. (a) Actual crack, (b) apparent crack deduced from raw image, and (c) inferred crack according to the proposed methodology for Case 4.

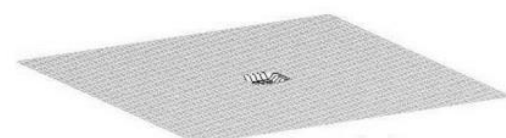

(a)

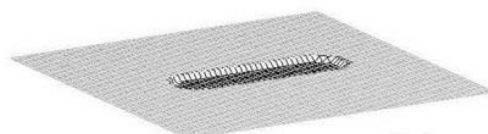

(b)

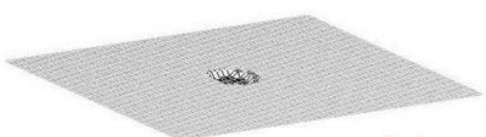

(c)

Figure 9. (a) Actual crack, (b) apparent crack deduced from raw image, and (c) inferred crack according to the proposed methodology for Case 5.

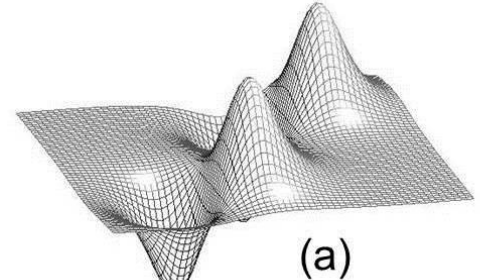

(a)

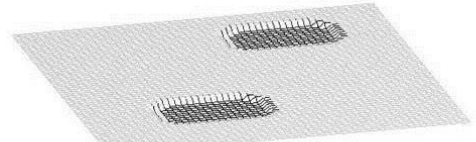

(c)

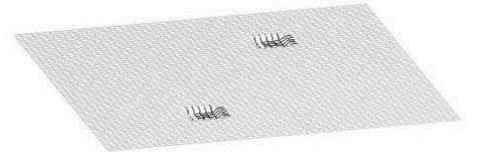

(b)

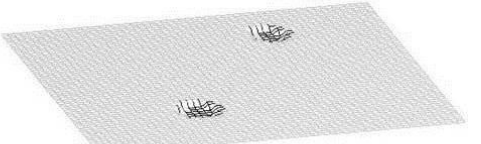

(d)

Figure 10. (a) Computed $B_{z}^{M}$, (b) actual cracks, (c) apparent cracks deduced from raw image, and (d) inferred cracks according to the proposed methodology for Case 6.

Figure 11 shows the device response $K_{z}$, as well as its reconstructed response using 2D FT, for Case 7 which corresponds to a sensor side length of $0.3 \mathrm{pu}$. Plots for $B_{z}^{M}$ computed in accordance with equation (3), actual crack, apparent crack deduced from raw images and inferred crack according to equation (5) are shown in Figure 12. Finally, device response $K_{z}$, as well as its reconstructed response using 2D FT, for Case 8 corresponding to a sensor side length of $0.5 \mathrm{pu}$ are given in Figure 13. On the other hand, Figure 14 demonstrates - for the same crack of Case 7 - the computed $B_{z}^{M}$ in accordance with equation (3), actual crack, apparent crack deduced from raw images and inferred crack using equation (5). 


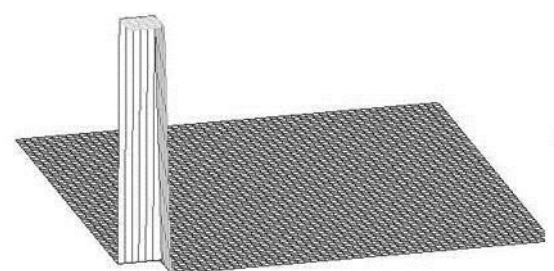

(a)

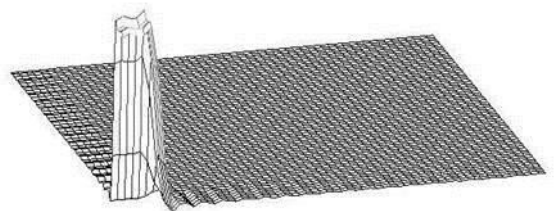

(b)

Figure 11. (a) The device response $K_{z}$ corresponding to Case 7 and (b) the reconstructed response using 2D FT harmonic coefficients.

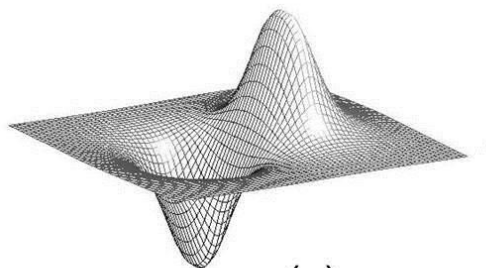

(a)

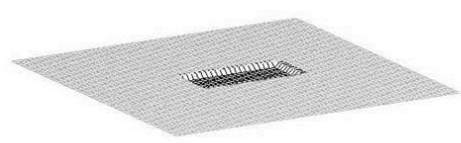

(c)

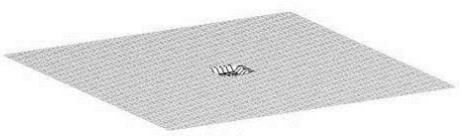

(b)

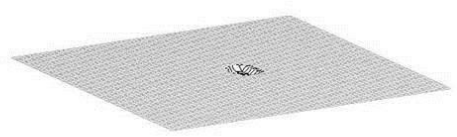

(d)

Figure 12. (a) Computed $B_{z}^{M}$, (b) actual cracks, (c) apparent cracks deduced from raw image, and (d) inferred cracks according to the proposed methodology for Case 7.

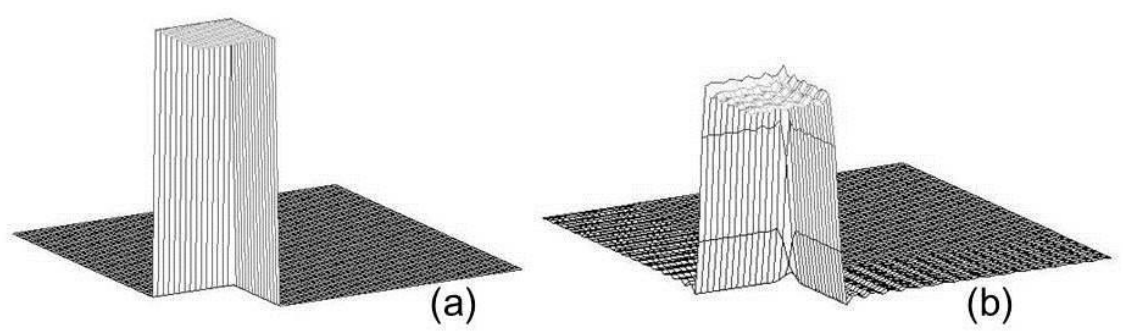

Figure 13. (a) The device response $K_{z}$ corresponding to Case 8 and (b) the reconstructed response using 2D FT harmonic coefficients.

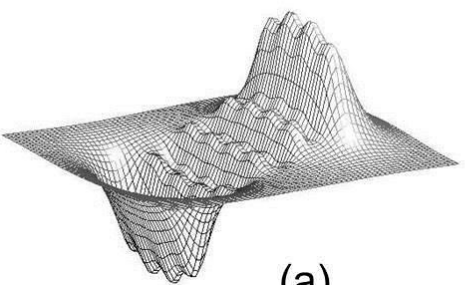

(a)

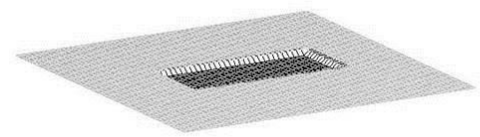

(c)

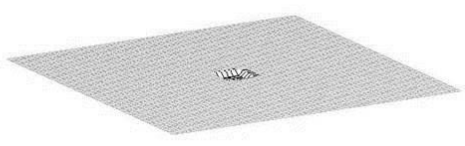

(b)

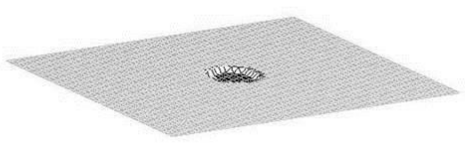

(d) 
Figure 14. (a) Computed $B_{z}^{M}$, (b) actual cracks, (c) apparent cracks deduced from raw image, and (d) inferred cracks according to the proposed methodology for Case 8. The above shown figures clearly highlight the ability of the proposed approach to cancel out the device response and, thus, reconstruct a very close three dimensional image of the assumed crack. Moreover, crack dimensions do not necessarily have to be in quanta of the FT discretization grid unlike for the case of using the wavelets approach (refer, for instance, to [9]).

\section{CONCLUSIONS}

It can be concluded from the presented analysis, simulations and results that the proposed approach may be well utilized to identify cracks whose dimensions are much smaller than sensing element dimensions and/or sensing height. Results also demonstrate that the proposed approach may also resolve the existence of multiple cracks. An important advantage of the proposed approach is its straight forward nature. Future extensions include the utilization of the approach in other related inverse problem and nondestructive testing techniques.

\section{REFERENCES}

[1] M. Oka and M. Enokizono, "A Detection of Backside Crack Using Rotational Magnetic Flux Sensor With Search Coils," IEEE Trans. Magn., vol. 33, pp. 4968-4970, 1996.

[2] A.A. Adly, "A Variable Frequency Electromagnetic Technique for Locating Embedded Cracks in Conducting Media," IEEE Trans. Magn., vol. 37, pp. 3800-3803, 2001.

[3] S.K. Abd-El-Hafiz and A.A. Adly, "Identification of Surface Cracks in Magnetic Bodies Using Wavelets on a Bounded Interval," Physica-B: Condensed Matter, vol. 406, pp. 280-286, 2011.

[4] Fenglian Niu, "Crack Detection of Power Line Based on Metal Magnetic Memory Nondestructive," TELKOMNIKA Indonesian Journal of Electrical Engineering, vol 12, pp. 7764-7771, 2014.

[5] R. Rajni, A. Kaur and A. Marwaha, "Complementary Split Ring Resonator based Sensor for Crack Detection," Int. Journal of Electrical and Computer Engineering (IJECE), vol. 5, pp. 1012-1017, 2015.

[6] I. Sebestyen and J. Paw, "Flaw Detection Using DC Magnetic Field Measurement," International Journal of Applied Electromagnetics and Mechanics, vol. 15, pp. 53-60, 2003.

[7] P. Giordano, J.M. Ittel, J. Cahouet and S. Daste, "Modelization of Cracks in Steel Subsea Structures: Application to The Optimization of a New Electromagnetic Detection Method," IEEE Trans. Magn., vol. 37, pp. 5408-5410, 1991.

[8] A.A. Adly and S.K. Abd-El-Hafiz, "A Wavelet Approach for The Identification of Surface Cracks Using Current Injection Perturbation," Journal of Applied Physics, vol. 111, pp. 07E316, 2012.

[9] S.K. Abd-El-Hafiz and A.A. Adly, "Three Dimensional Identification of Crack Location in Conducting Slabs Using Wavelets," IEEE Trans. Magn., vol. 49, pp. 3472-3475, 2013.

[10] I.D. Mayergoyz, A.A. Adly, R.D. Gomez, and E.R. Burke, "Magnetization Image Reconstruction from Magnetic Force Scanning Tunneling Microscopy Images,” Journal of Applied Physics, vol. 73, pp. 5799-5801, 1993.

[11] R. Madabhushi, R.D. Gomez, E.R. Burke, and I.D. Mayergoyz, "Magnetic Biasing and MFM Image Reconstruction," IEEE Trans. Magn., vol. 32, pp. 4147-4149, 1996.

[12] A.A. Adly, M.M. Abdel-Aziz and N.S. Hosny, "Deducing Local ELF Field Values From Large Sense Coil Fluxmeter Measurements,” Journal of Applied Physics, vol. 105, pp. 07E721, 2009.

\section{BIOGRAPHIES OF AUTHORS}

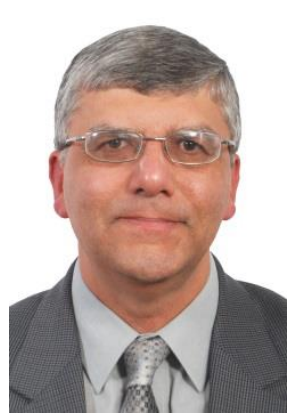

Amr A. Adly (IEEE Fellow) received the B.Sc. and M.S. degrees in Elect. Eng. from Cairo University (CU) in 1984 and 1986, respectively, and the Ph.D. degree in Elect. Eng. from the University of Maryland (UMD), College Park, USA, in 1992. He also worked as a Magnetic Measurement Instrumentation Senior Scientist at LDJ Electronics, Michigan, USA, during 1993 1994. Since 1994, he has been a faculty member at the Dept. of Elect. Power and Machines CU. He also worked in the US as a Visiting Research Prof. at UMD, during the summers of 19962000. He worked in the period 2003-2004 as a consultant to the UNESCO Cairo office and in 2006 as an expert to the EU Commission. He also established and directed the R\&D Administration at the Egyptian Industrial Modernization Center in the period 2006-2007. He served as the CU's Faculty of Engineering Vice Dean in the period 2010-2014. In the period 2014-2015 he was been appointed as the Executive Director of the Science and Technology Development Fund (STDF). Since January 2016 he has been appointed as Cairo University Vice President for Graduate Studies and Research. Prof. Adly has published more than 125 reviewed papers, holds one U.S. patent, acted as the PI of several international and has been awarded the 1994 Egyptian State Encouragement Prize, the 2002 Shoman Foundation Young Arab Scientist Prize and the 2006 Egyptian State Excellence Prize. He has been promoted to IEEE Fellow 
status in 2011 and is currently serving in the Editorial Board of IEEE Transactions on Magnetics and Elsevier's Journal of Advanced Research.

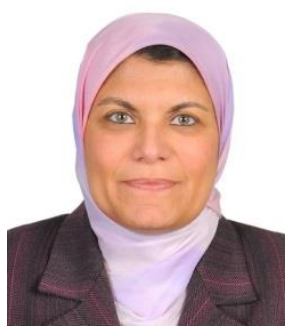

Salwa K. Abd-El-Hafiz (IEEE Senior Member) received the B.Sc. degree in Electronics and Communication Engineering from Cairo University (CU) in 1986 and the M.S. and Ph.D. degrees in Computer Science from the University of Maryland (UMD), College Park, Maryland, USA, in 1990 and 1994, respectively. Since 1994, she has been working as a Faculty Member in the Engineering Mathematics and Physics Department CU, and has been promoted to a Full Professor in the same department in 2004. In the period 2014-2016, she has been working as the Director of the Technical Center for Career Development, CU. Since August 2016, she was appointed as the Chairman of the Engineering Mathematics and Physics Department CU. She coauthored one book, contributed one chapter to another book and published more than 60 refereed papers. Prof. Abd-El-Hafiz is a recipient of the 2001 Egyptian State Encouragement Prize in Engineering Sciences, recipient of the 2012 National Publications Excellence Award from the Egyptian Ministry of Higher Education, recipient of the 2014 African Union Kwame Nkrumah Regional Scientific Award for Women in basic science, technology and innovation, recipient of several international publications awards from Cairo University and an IEEE Senior Member. 